\title{
Exploring the role of neurogenic pathway-linked cholecystokinin release in remote preconditioning-induced cardioprotection ${ }^{1}$
}

\author{
Huilian Li' (D) , Cuilan An" \\ ' BS, Medical Record Room, Central Hospital Affiliated to Shandong First Medical University, Jinan, China. \\ Technical procedures, acquisition and analysis of data. \\ " BS, Department of Health Care, Central Hospital Affiliated to Shandong First Medical University, Jinan, China. \\ Manuscript preparation and writing.
}

\begin{abstract}
Purpose: The current study explored the involvement of neurogenic pathway-linked cholecystokinin (CCK) release in RIP-induced cardioprotection in rats.

Methods: Male Wistar rats were subjected to four cycles of alternate episodes of ischemia and reperfusion (five min each) to induce RIP. Thereafter, the hearts were subjected to global ischemia and reperfusion ex vivo. The myocardial damage was assessed by quantifying the levels of heartspecific biochemicals i.e. LDH-1, CK-MB and cTnT. Apoptotic cell injury was assessed by measuring the levels of caspase-3 and $\mathrm{Bcl}-2$. The levels of CCK were measured in the plasma following RIP.

Results: Exposure to RIP significantly increased the plasma levels of CCK and attenuated IR-induced myocardial injury. Administration of CCK antagonist, proglumide significantly attenuated RIP-induced cardioprotection. Administration of hexamethonium, a ganglion blocker, abolished RIP-induced increase in plasma CCK levels and cardioprotective effects. Exogenous delivery of CCK-8 restored the effects of RIP in hexamethonium treated animals.

Conclusion: RIP activates the neurogenic pathway that may increase the plasma levels of CCK, which may act on the heart-localized CCK receptors to produce cardioprotection against I/R injury.
\end{abstract}

Key words: Cholecystokinin. Remote Preconditioning. Ischemia. Apoptosis. Neurogenic Pathway. Rats. 


\section{- Introduction}

Ischemia-Reperfusion (I/R)-induced myocardial injury is one of the leading causes of mortality and morbidity worldwide ${ }^{1}$. However, there are limited interventions to successfully manage I/R-induced myocardial injury. Remote preconditioning (RIP) is one of the interventions that may have been found to reduce the extent of myocardial injury. In this intervention, exposure of an organ (other than heart) to alternate, short cycles of ischemia and reperfusion confers protection to the heart against sustained I/R injury ${ }^{2}$. The therapeutic utility of RIP has been reported in studies pertaining to animals and humans ${ }^{3,4}$. However, the mechanisms involved in RIP-induced cardioprotection are still not fully explored.

Cholecystokinin (CCK) is a neuropeptide that affects growth by regulating appetite. It is synthesized and secreted by enteroendocrine cells in the duodenum ${ }^{5}$. CCK produces actions through CCK-1 and CCK-2 receptors, which are present on different tissues, including heart ${ }^{6,7}$. Apart from regulating the local actions in the gastrointestinal tract, studies have documented that this neuropeptide may also produce diverse actions including in the pathophysiology of cancer, behavioral disorders, pain and memory disorders ${ }^{8}{ }^{10}$. CCK also regulates the functions of heart in physiological and pathological conditions ${ }^{11}{ }^{13}$. Considering the presence of CCK receptors on the heart and its role in regulating cardiac functions including ischemia, it was hypothesized that there may be a possible role of CCK in RIP-induced cardioprotection and its release during RIP may be linked to a neural pathway. Therefore, the current investigation explored the involvement of neurogenic pathwaylinked cholecystokinin (CCK) release in RIP-induced cardioprotection in rats

\section{- Methods}

The experiments were approved by the Central Hospital Affiliated to Shandong First Medical University, under the number: 2020-0618-01. All experiments were performed as per institutional ethical guidelines in the Research Center of the Central Hospital Affiliated to Shandong First Medical University, Jinan, China.

In this investigation, Wistar albino rats of weight 210-240g were used. The doses of CCK- $8^{14}$, Proglumide ${ }^{15}$, heaxmethonium $^{16}$ were selected as per literature reports. The kits for the quantification of lactate dehydrogenase 1 (LDH-1), MB isoform of creatine kinase (CK-MB), cardiac troponins (cTnT), Bcl-2, caspase 3 and CCK were procured from MyBioSource, Inc. San Diego, CA USA.

\section{Remote ischemic preconditioning (RIP)}

After anesthetizing the rats with thiopental sodium (45 mg/kg), a neonatal blood pressure cuff was tied on one of the hind limbs (left). Thereafter, the inflation (up to $150 \mathrm{~mm}$ of $\mathrm{Hg}$ ) and deflation (zero pressure) of cuff was done in an alternate manner to stop (ischemia) and resume (reperfusion) blood supply to the hind limb, respectively. The period of hind limb ischemia and reperfusion constituted five minutes and the hind limb was subjected to four such transient, alternate cycles. After completion of RIP protocol (40 min), the animals were sacrificed and hearts were isolated ${ }^{17}$.

\section{Ischemia-reperfusion injury}

The isolated hearts were mounted on the Langendorff system and perfused with perfused with Kreb's Henseleit solution at $37^{\circ} \mathrm{C}$. The inflow of physiological solution was stopped for 30 minutes to induce global ischemia and afterwards, the flow of $\mathrm{KH}$ was reinstituted for 120 minutes to establish reperfusion ${ }^{18,19}$.

\section{Assessment of heart-specific biochemicals of heart}

In this investigation, IR injury to heart was quantified using three different heart-specific biochemicals i.e. LDH1, CK-MB, and cTnT. The presence of these biochemicals was assessed in the coronary effluent before subjecting to global ischemia and after instituting reperfusion. To determine the LDH-1 activity, guanidine thiocyanate was added to suppress enzymatic activity of other isoforms of $\mathrm{LDH}$, including LDH-2, LDH-3, LDH-4, and LDH-5. After that, lithium L-lactate and $\mathrm{NAD}^{+}$were added in the sample (containing LDH) to yield pyruvic acid and $\mathrm{NADH}$, and absorbance was measured at $340 \mathrm{~nm}$ wavelength.

The determination of $\mathrm{CK}-\mathrm{MB}$ involved the addition of $A D P$ and creatine kinase in the coronary effluent samples (containing CK-MB) to obtain ATP, which was allowed to react with glucose in the presence of hexokinase. After that, NADP ${ }^{+}$and glucose-6-phosphate dehydrogenase were added in the reaction mixture that led to the production of NADPH and its absorbance was determined at $340 \mathrm{~nm}$ to quantify enzymatic activity.

CTnT was quantified by the sandwich assay-based ELISA kit. The wells of microplate were coated with an antibody specific to cTnT (also called capture antibody). Thereafter, the coronary effluent sample (containing cTnT) was added into the wells that allowed the binding of cTnT to capture antibody. It was followed by the addition of a biotin-conjugated antibody (detection reagent $A$ ) specific to cTnT. After washing, avidin conjugated to horseradish peroxidase (HRP) (detection reagent B) was made complex with the biotin-conjugated antibody. After 
washing, a substrate for HRP i.e. tetramethylbenzidine was added and this yielded the color development, which was measured at 450nm.

\section{Assessment of apoptotic cell injury}

The assessment of apoptotic cell death in the heart was done by quantifying the levels of caspase 3 and bcl-2 in the heart homogenates. These apoptotic parameters were measured using ELISA kits.

\section{Quantification of plasma CCK levels}

The blood was isolated at the time of sacrificing the rats and plasma levels of CCK were measured using ELISA kits.

\section{Study design}

In this investigation, seven groups were employed and eight animals were employed in each group. These groups included (i) I/R injury (ii) RIP (iii) Proglumide (10 mg/kg i.p.) in RIP (iv) Proglumide $(20 \mathrm{mg} / \mathrm{kg}$ i.p.) in RIP (v) Hexamethonium (20 mg/kg i.p.) in RIP (vi) CCK-8 $(20 \mu \mathrm{g} / \mathrm{kg}$ i.v) \& hexamethonium in RIP (vii) CCK-8 $(20 \mu \mathrm{g} / \mathrm{kg}$ i.v) \& hexamethonium in RIP

\section{Statistics}

Mean \pm standard deviation (S.D.) was used to represent the data of this study. The results of $L D H-1$,
CK-MB, and CTnT were analyzed using two way repeated measure ANOVA. The data of all other parameters were analyzed using One-way ANOVA. These tests were followed by Tukey's post hoc test. The statistical significance was fixed at $p<0.05$.

\section{Results}

\section{Decrease in I/R-induced myocardial injury in response to $R I P$}

The levels of heart-specific biochemicals i.e. LDH-1 (Fig. 1), CK-MB (Fig. 2) and CTnT (Fig. 3) were increased in response to ischemia-reperfusion injury. Indeed, their levels were very high during the reperfusion phase as compared to the basal state i.e. before initiating global ischemia to the heart suggesting significant myocardial injury in I/R-subjected rats. Moreover, a marked rise in the parameters of apoptosis i.e. augmentation of caspase-3 (proapoptotic), (Fig. 4) and reduction in $\mathrm{Bcl}-$ 2 (antiapoptotic) (Fig. 5) was observed in the heart homogenates, suggesting the significant apoptotic cell injury in I/R-subjected rats. However, in RIP-subjected rats, there was a marked decrease in the release of heart-specific biochemicals from the heart following thirty minutes of ischemia (Figs. 1 to 3). Furthermore, RIP decreased the levels of caspase- 3 and increased the levels of $\mathrm{Bcl}-2$ suggesting the decrease in apoptotic cell injury.

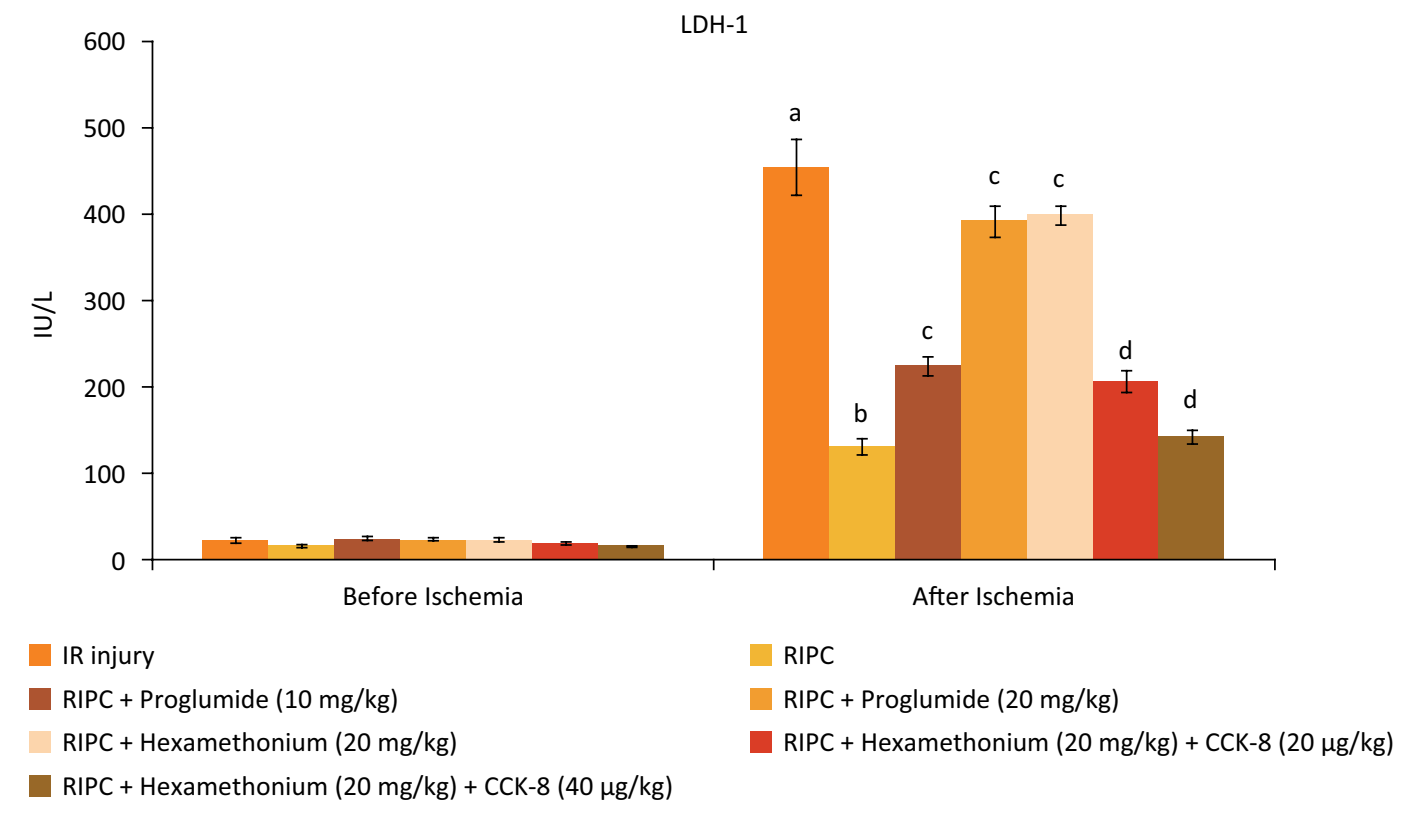

Figure 1 - Influence of various treatments on LDH-1 levels. $\mathrm{a}=p<0.05$ vs. IR injury, before ischemia; $\mathrm{b}=p<0.05$ vs. IR injury, after ischemia; $c=p<0.05$ vs. RIP; $d=p<0.05$ RIP + hexamethonium. 


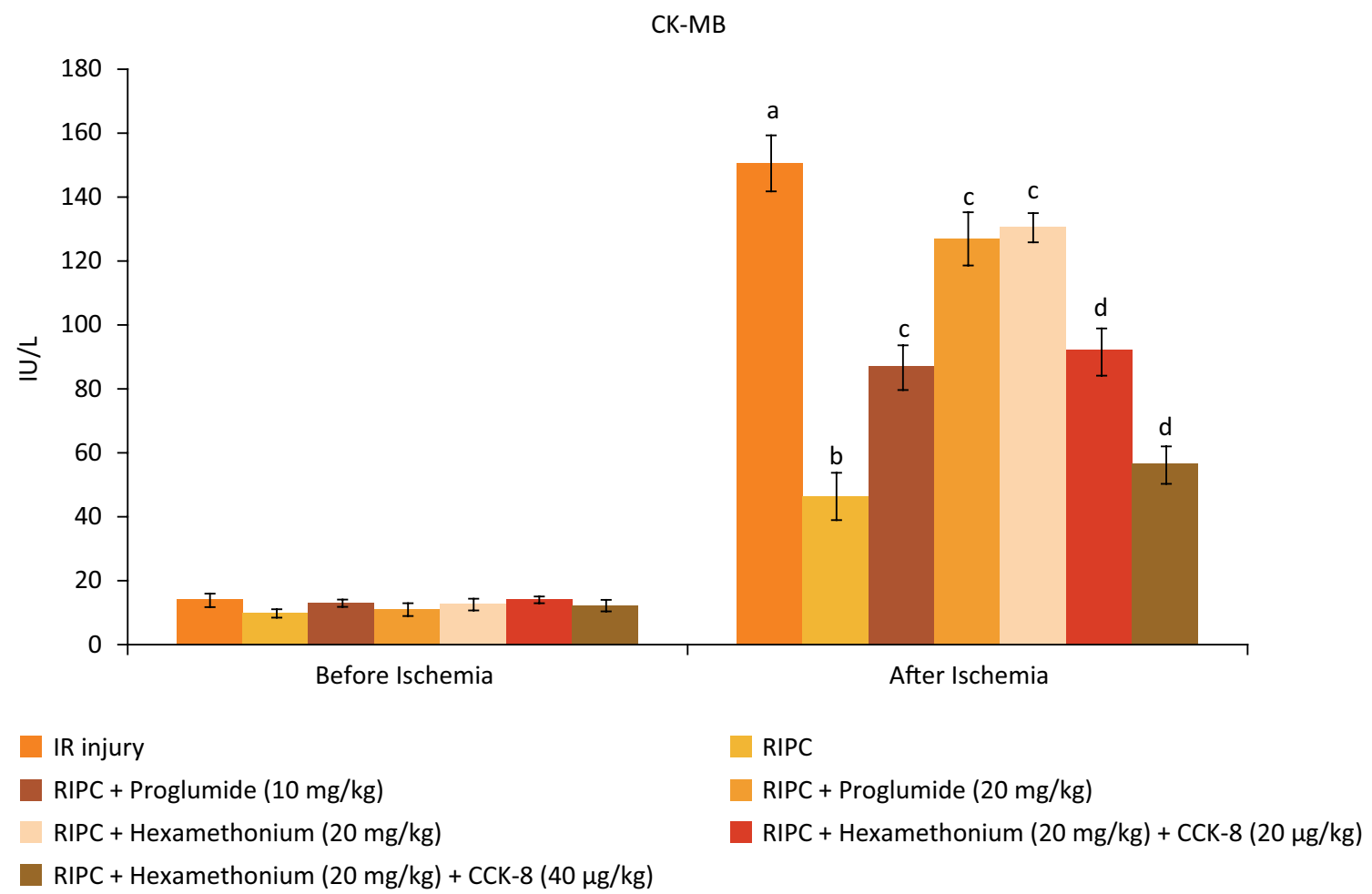

Figure 2 - Influence of various treatments on CK-MB levels. $\mathrm{a}=p<0.05$ vs. IR injury, before ischemia; $\mathrm{b}=p<0.05$ vs. IR injury, after ischemia; $c=p<0.05$ vs. RIP; $d=p<0.05$ RIP + hexamethonium.

CTnT

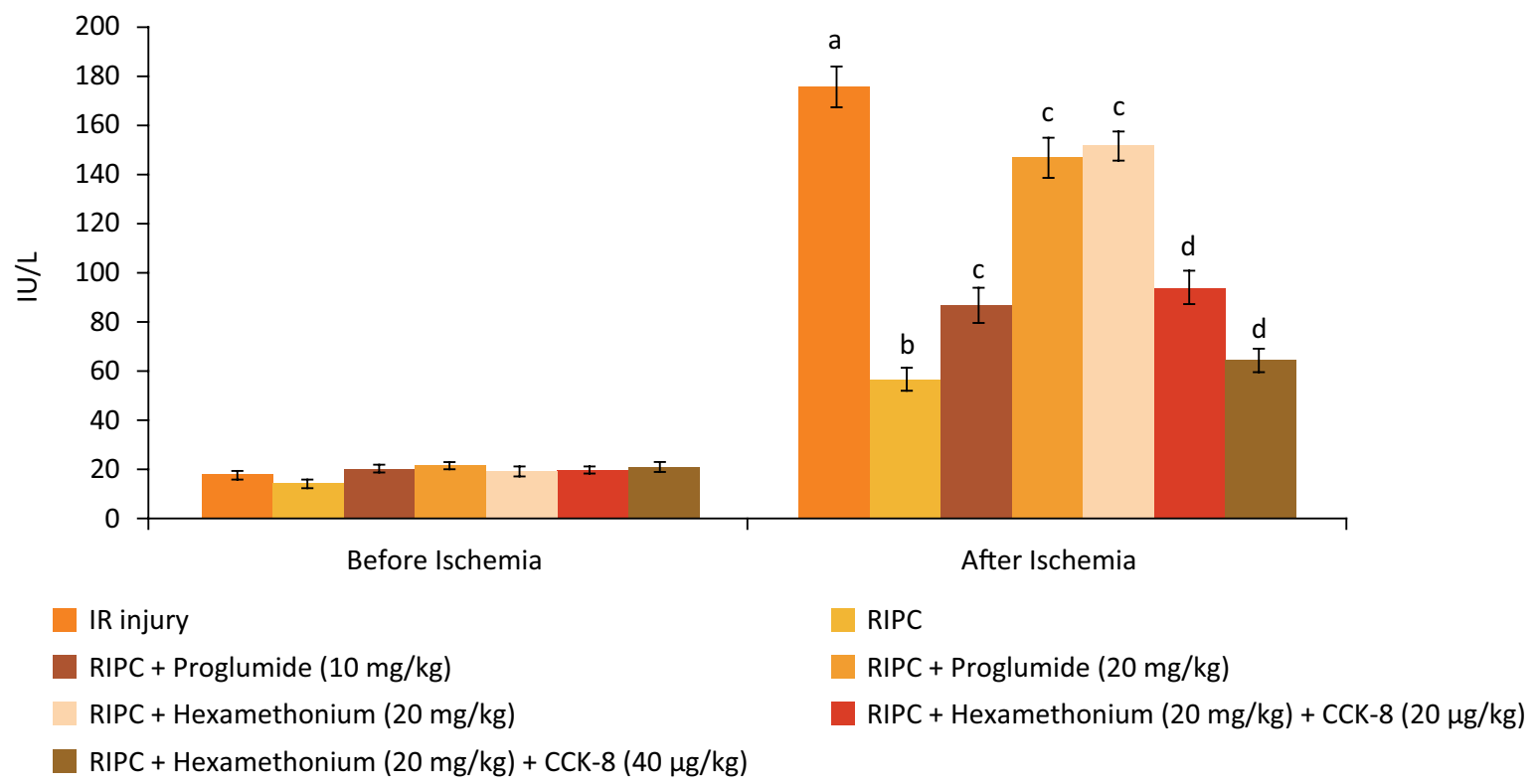

Figure 3 - Influence of various treatments on cTnT levels. $a=p<0.05$ vs. IR injury, before ischemia; $b=p<0.05$ vs. IR injury, after ischemia; $c=p<0.05$ vs. RIP; $d=p<0.05$ RIP + hexamethonium. 
hexamethonium significantly abolished RIP-mediated increase in the CCK levels. However, exogenous delivery of CCK-8 restored the plasma CCK levels in hexamethonium treated RIP rats. Nevertheless, CCK antagonist did not modulate RIP-mediated increase in CCK levels.

\section{- Discussion}

In this current investigation, RIP provided cardioprotection against I/R-induced injury as there was significant reduction in the release of heartspecific biochemicals including LDH-1, CK-MB and cTnT. Moreover, RIP also normalized the parameters of apoptosis in terms of reduction in proapoptotic, caspase- 3 and augmentation of antiapoptotic, $\mathrm{Bcl}-2$. This suggests that RIP also attenuated I/R-induced apoptotic cell injury. There have been studies suggesting that RIP attenuates I/R-induced myocardial injury ${ }^{20,21}$.

In this current investigation, RIP-induced cardioprotective effects were also associated with a marked increase in CCK levels. CCK is a neuropeptide that helps in regulating appetite and growth. Apart from it, studies have shown its key role in cancer ${ }^{8}$, memory ${ }^{9}$, irritable bowel syndrome ${ }^{20}$, pain ${ }^{10}$ and anxiety ${ }^{21}$. Apart from it, CCK has a key role in regulating cardiovascular functions. Both, CCK1 and CCK2 receptors are expressed on the heart ${ }^{6,7}$. Studies have shown that CCK may regulate cardiac function ${ }^{11,12}$. Moreover, the marked alterations in the CCK receptors density have been documented in the ischemic heart ${ }^{13}$. Accordingly, it can be hypothesized that RIP augments the levels of CCK in the circulation that travel to the heart to produce cardioprotection against $I / R$ injury. To verify the role of CCK in RIP-induced cardioprotection, non-selective CCK antagonist (CCK1 and CCK2 receptor antagonist) i.e. proglumide was administered prior to RIP stimulus. Administration of proglumide significantly attenuated RIP-induced cardioprotection suggesting that CCK may be critically involved in RIP-induced cardioprotection. To best of our knowledge, it is the first study describing the key role of CCK in RIP-induced cardioprotection against I/R injury.

To further explore the mechanisms involved in RIP-induced increase in CCK release and cardioprotection, hexamethonium (ganglion blocker) was co-administered in RIP-subjected rats. Administration of hexamethonium significantly attenuated RIP-induced increase in CCK release. Moreover, hexamethonium also abolished RIP-induced cardioprotection. There have been studies suggesting that RIP may trigger the activation of neurogenic pathway to induce cardioprotection ${ }^{22}$. Based on the results of this investigation, it seems that RIP may trigger a neurogenic pathway to augment the CCK levels in circulation. In the other words, RIP-induced increase in CCK levels is dependent on the activation of a neurogenic pathway. Exogenous delivery of CCK agonist i.e. CCK-8 significantly restored the cardioprotective effects in hexamethonium-treated RIPC rats, which further suggests the key role of CCK in RIP-induced cardioprotection in I/R-subjected rats. There have been studies documenting the individual role of humoral factors $^{23}$ and the neurogenic pathway ${ }^{16,22}$ in RIPinduced cardioprotection. However, the present study attempts to correlate the two different pathways and it may be proposed that the neurogenic and humoral pathways are not totally independent. Rather, these pathways are interlinked and our study proposes that the activation of the neurogenic pathway triggers the release of CCK (as humoral factor), which may act on the heart to produce cardioprotection.

\section{- Conclusion}

There is a key role of CCK in RIP-induced cardioprotection in I/R-subjected rats. It may be possible that RIP stimulates the neurogenic pathway leading to increase in the CCK levels, which may act on heart-localized CCK1 and CCK2 receptors to produce cardioprotection against I/R injury.

\section{- References}

1. Ibáñez B, Heusch G, Ovize $M$, Van de Werf F. Evolving therapies for myocardial ischemia/reperfusion injury. J Am Coll Cardiol. 2015;65(14):1454-71. doi: 10.1016/j. jacc.2015.02.032.

2. Tsibulnikov SY, Maslov LN, Gorbunov AS, Voronkov NS, Boshchenko AA, Popov SV, Prokudina ES, Singh N, Downey JM. A review of humoral factors in remote preconditioning of the heart. J Cardiovasc Pharmacol Ther. 2019;24(5):40321. doi: $10.1177 / 1074248419841632$.

3. Healy DA, Walsh SR. Remote preconditioning and vascular surgery. J Cardiovasc Pharmacol Ther. 2017;22(4):316-20. doi: $10.1177 / 1074248417702892$.

4. Donato M, Evelson P, Gelpi RJ. Protecting the heart from ischemia/reperfusion injury: an update on remote ischemic preconditioning and postconditioning. Curr Opin Cardiol. 2017;32(6):784-90. doi: 10.1097/HCO.0000000000000447.

5. Owyang C. Physiological mechanisms of cholecystokinin action on pancreatic secretion. Am J Physiol. 1996;271(1 Pt 1):G1-7. doi: 10.1152/ajpgi.1996.271.1.G1.

6. Zhao X-Y, Ling Y-L, Li Y-G, Meng A-H, Xing H-Y. Cholecystokinin octapeptide improves cardiac function by activating cholecystokinin octapeptide receptor in endotoxic shock rats. World J Gastroenterol. 2005;11(22):3405-10. doi: 10.3748/wjg.v11.i22.3405 
7. Yang $X$, Yue R, Zhang J, Zhang $X$, Liu $Y$, Chen C, Wang $X$, Luo $H$, Wang WE, Chen $X$, Wang $H J$, Jose PA, Wang $H$, Zeng $C$. Gastrin protects against myocardial ischemia/reperfusion injury via activation of RISK (Reperfusion Injury Salvage Kinase) and SAFE (Survivor Activating Factor Enhancement) pathways. J Am Heart Assoc. 2018;7(14):e005171. doi: 10.1161/JAHA.116.005171.

8. Lloyd KA, Parsons BN, Burkitt MD, Moore AR, Papoutsopoulou S, Boyce M, Duckworth CA, Exarchou K, Howes N, Rainbow L, Fang Y, Oxvig C, Dodd S, Varro A, Hall N, Pritchard DM. Netazepide inhibits expression of pappalysin 2 in type 1 gastric neuroendocrine tumors. Cell Mol Gastroenterol Hepatol. 2020;10(1):113-32. doi: 10.1016/j.jcmgh.2020.01.010.

9. Nguyen R, Venkatesan S, Binko M, Bang JY, Cajanding JD, Briggs C, Sargin D, Imayoshi I, Lambe EK, Kim JC. Cholecystokinin-expressing interneurons of the medial prefrontal cortex mediate working memory retrieval. J Neurosci. 2020;40(11):2314-31. doi: 10.1523/ JNEUROSCI.1919-19.2020.

10. Keppel Hesselink JM. Rediscovery of ceruletide, a CCK agonist, as an analgesic drug. J Pain Res. 2020;13:123-30. doi: 10.2147/JPR.S232714.eCollection 2020.

11. Seth $H$, Gräns A, Axelsson M. Cholecystokinin as a regulator of cardiac function and postprandial gastrointestinal blood flow in rainbow trout (Oncorhynchus mykiss). Am J Physiol Regul Integr Comp Physiol. 2010;298(5):R1240-8. doi: 10.1152/ajpregu.00781.2009.

12. Goetze JP, Rehfeld JF, Alehagen U. Cholecystokinin in plasma predicts cardiovascular mortality in elderly females. Int J Cardiol. 2016;209:37-41. doi: 10.1016/j. ijcard.2016.02.038.

13. Dong X, Wang C, Zhang J, Wang S, Li H, Kang Y, Tian S, $\mathrm{Fu}$ L. Cholecystokinin expression in the development of postinfarction heart failure. Cell Physiol Biochem. 2017;43(6):2479-88. doi: 10.1159/000484454.

14. Hashimoto $H$, Onaka $T$, Kawasaki $M$, Chen $L$, Mera $T$, Soya A, Saito T, Fujihara H, Sei H, Morita Y, Ueta Y. Effects of cholecystokinin (CCK)-8 on hypothalamic oxytocinsecreting neurons in rats lacking CCK-A receptor. Auton Neurosci. 2005;121(1-2):16-25. doi: 10.1016/j. autneu.2005.05.002.

15. Inoue S, Johanek LM, Sluka KA. Lack of analgesic synergy of the cholecystokinin receptor antagonist proglumide and spinal cord stimulation for the treatment of neuropathic pain in rats. Neuromodulation. 2017;20(6):534-42. doi: 10.1111/ner.12601.

16. Singh $H$, Kumar $M$, Singh $N$, Jaggi AS. Late phases of cardioprotection during remote ischemic preconditioning and adenosine preconditioning involve activation of neurogenic pathway. J Cardiovasc Pharmacol. 2019;73(2):63-9. doi: 10.1097/FJC.0000000000000634.

17. Singh A, Randhawa PK, Bali A, Singh N, Jaggi AS. Exploring the role of TRPV and CGRP in adenosine preconditioning and remote hind limb preconditioninginduced cardioprotection in rats. Cardiovasc Drugs Ther. 2017;31(2):133-43. doi: 10.1007/s10557-017-6716-3.

18. Torregroza C, Feige K, Schneider L, Bunte $S$, Stroethoff $M$, Heinen A, Hollmann MW, Huhn R, Raupach A. Influence of hyperglycemia on dexmedetomidine-induced cardioprotection in the isolated perfused rat heart. J Clin Med. 2020;9(5):1445. doi: 10.3390/jcm9051445.

19. Billah M, Ridiandries A, Allahwala UK, Mudaliar H, Dona A, Hunyor S, Khachigian LM, Bhindi R. Remote ischemic preconditioning induces cardioprotective autophagy and signals through the IL-6-dependent JAK-STAT pathway. Int J Mol Sci. 2020;21(5):1692. doi: 10.3390/ ijms21051692.

20. Qin G, Zhang Y, Yao SK. Serotonin transporter and cholecystokinin in diarrhea-predominant irritable bowel syndrome: associations with abdominal pain, visceral hypersensitivity and psychological performance. World J Clin Cases. 2020;8(9):1632-41. doi: 10.12998/wjcc. v8.i9.1632.

21. Matsuda K, Yoshida D, Sachuriga null, Watanabe K, Yokobori E, Konno N, Nakamachi T. Effect of intracerebroventricular administration of two molecular forms of sulfated CCK octapeptide on anxiety-like behavior in the zebrafish danio rerio. Peptides. 2020;130:170330. doi: 10.1016/j. peptides.2020.170330.

22. Aulakh AS, Randhawa PK, Singh N, Jaggi AS. Neurogenic pathways in remote ischemic preconditioning induced cardioprotection: Evidences and possible mechanisms. Korean J Physiol Pharmacol. 2017;21(2):145-52. doi: 10.4196/kjpp.2017.21.2.145.

23. Tsibulnikov SY, Maslov LN, Gorbunov AS, Voronkov NS, Boshchenko AA, Popov SV, Prokudina ES, Singh N, Downey JM. A review of humoral factors in remote preconditioning of the heart. J Cardiovasc Pharmacol Ther. 2019;24(5):403-21. doi: 10.1177/1074248419841632. 


\section{Correspondence:}

\section{Cuilan An}

Department of Health Care

Central Hospital Affiliated to Shandong First Medical

University

Jinan, 25001 China

Phone: +86-151-12009654

aclwmh@sina.com

Received: May 13, 2020

Review: July 10, 2020

Accepted: Aug 12, 2020
Conflict of interest: none

Financial source: none

This is an Open Access article distributed under the terms of the Creative Commons Attribution License, which permits unrestricted use, distribution,
and reproduction in any medium, provided the original work is properly cited.

${ }^{1}$ Research performed at Department of Health Care, Central Hospital Affiliated to Shandong First Medical University, Jinan, China. and reproduction in any medium, provided the original work is properly cited. 